\title{
EMISSION FROM DUST NEAR HIGH-LATITUDE STARS
}

\author{
J. Murthy \\ National Research Councill \\ Resident Research Associate \\ Lab. for Astronomy and Solar Physics \\ Goddard Space Flight Center \\ Greenbelt, Maryland 20771 USA \\ M. W. Werner and H. J. Walker \\ NASA AMES Research Center \\ MS 245-6 \\ Moffett Field, California 94035 USA
}

\author{
R. C. Henry, R. A. Kimble, and \\ J. B. Wofford \\ Dept. of Physics and Astronomy \\ Johns Hopkins University \\ Baltimore, Maryland 21218 USA
}

\section{INTRODUCTION}

The spatial distribution of interstellar dust is clearly important in understanding not only galactic dynamics but also the processing of the dust and the interstellar medium in general. Probably the best spectral region for investigating interstellar dust is the infrared (IR), where the cool dust is likely to radiate. Indeed, one of the most prominent features of the IRAS sky is the ubiquitous cirrus emission, thought to be due to interstellar dust heated by the interstellar radiation field (ISRF), seen at 60 and $100 \mu \mathrm{m}$ (Low 1984). However, it is difficult to use the cirrus to probe the dust distribution, both because we have no depth information and also because the cirrus, due to its low temperatures $(-20 \mathrm{~K})$, is a probe of high-density dust regions. A far more sensitive search could be made if the dust were hotter, that is, in the presence of a greater ultraviolet (UV) flux. We have made use of this fact to search for dust in the vicinity of hot, bright stars, where even a small amount of dust will dominate the total emission along that line of sight.

\section{DATA ANALYSIS}

The zodiacal light is a major contaminant in both the $60 \mu \mathrm{m}$ and the $100 \mu \mathrm{m}$ IRAS Sky Flux plates, and the first step in any procedure must be to remove it. We have made two assumptions in attempting to do this: (a) the $25 \mu \mathrm{m}$ plate is composed almost entirely of zodiacal light, except for a few isolated regions; and (b) the brightness of the zodiacal light in the two long-wavelength plates is a constant multiple of the brightness in the $25 \mu \mathrm{m}$ plate. We estimated these multiples by plotting the $60 / 25 \mu \mathrm{m}$ and the $100 / 25 \mu \mathrm{m}$ ratios and by fitting a baseline to them. We then fit the $25 \mu \mathrm{m}$ image in the region of interest by a quadratic and subtracted this, multiplied by the appropriate constant of proportionality, from the 60 and 100 $\mu \mathrm{m}$ plates, respectively.

A major problem with this procedure is that the ratio of the 60 or $100 \mu \mathrm{m}$ plate to the 25 $\mu \mathrm{m}$ plate does not appear to be constant over the plate for many regions, so it is difficult to establish a baseline. The ratio we select for a given plate is based on the lowest of these ratios and therefore we always underestimate the zodiacal correction and may leave residuals on the order of $2 \mathrm{MJy} \mathrm{sr}^{-1}$. Although this is not important where there is a significant amount of cirrus or for hot stars, for cool stars in a cirrus-free region it may critically limit the quality of our results. 
After the zodiacal light has been removed, our first-stage procedure is to place limits on the amount of dust that can be near a star. We use models for the stellar emission and for the optical properties of the dust to derive the 60 and $100 \mu \mathrm{m}$ emission from the dust within a given distance of the star. We then reverse the procedure to place limits on the amount of dust that can be within that distance of the star by using the 60 and $100 \mu \mathrm{m}$ IRAS Sky Flux plates.

\section{RESULTS AND DISCUSSION}

We have processed eight stars and our results are summarized in Table 1.

TABLE 1. Results

\begin{tabular}{|c|c|c|c|c|c|c|}
\hline Star & $l$ & $b$ & Sp Type & Peak $^{a}$ & $\mathrm{D}(1 \mathrm{pc})^{b}$ & $\mathrm{D}(2 \mathrm{pc})^{c}$ \\
\hline$\beta$ Cep & 108 & 14 & B2III & no & 0.48 & 1.3 \\
\hline$\delta \mathrm{Cet}$ & 171 & -52 & B2IV & no & 1.9 & 3.2 \\
\hline$\eta$ Нуа & 224 & 27 & B3V & no & 4.4 & 6.8 \\
\hline$\alpha$ Vir & 317 & 51 & B1V & yes & 0.86 & 0.95 \\
\hline$\omega$ Sco & 351 & 22 & BOV & small & 2.5 & 5.2 \\
\hline$\checkmark$ Sco & 353 & 22 & B1V & yes & 14.9 & 38.3 \\
\hline$\beta$ Sco & 354 & 23 & BOV & no & 0.14 & 0.32 \\
\hline$\delta$ Sco & 355 & 22 & B2IV & yes & 2.6 & 3.6 \\
\hline
\end{tabular}

Even in this preliminary work, we have placed some very interesting limits for the amount of dust (expressed in terms of $\mathrm{H}$ I density) near a few of our observed stars, such as the 0.32 $\mathrm{cm}^{-3}$ within $2 \mathrm{pc}$ of $\beta$ Sco. Further and more profound interpretation will have to await the analysis of a greater number of stars (work now in progress).

\section{CONCLUSIONS AND FURTHER WORK}

We have demonstrated that we can use the IRAS Sky Flux plates to place rather stringent limits on the amount of dust near several hot stars. We plan to explore the regions around a large number of hot stars by using this procedure, which has been set up to require limited human interaction. At the same time, our program is very modular, which allows us to replace any part, such as our zodiacal light subtraction, without much effort.

Concurrently with this global effort, we also plan to model in some detail the dust in individual regions, a task that may prove impossible to automate. Thus, our final goal is to explore the global characteristics of the dust and to model the three-dimensional distribution in selected areas.

\section{REFERENCES}

Low, F. J., et al. 1984, Ap. J. (Letters), 278, L19. 